Puesta al día en Medicina Intensiva:

Neurointensivismo

\title{
Tratamiento endovascular del vasospasmo cerebral inducido por hemorragia subaracnoidea aneurismática
}

\author{
PEDRO P. ALCÁZAR ${ }^{\mathrm{a}}$, ALEJANDRO GONZÁLEZ ${ }^{\mathrm{b}}$ Y ANTONIO ROMANCE ${ }^{\mathrm{c}}$ \\ aServicio de Radiología. Unidad de Neurorradiología Intervencionista. Hospital Universitario Virgen de las Nieves. \\ Granada. España. \\ 'Servicio de Radiología. Unidad de Neurorradiología Intervencionista. Hospital Universitario Virgen del Rocío. \\ Sevilla. España. \\ ‘Servicio de Radiología. Unidad de Neurorradiología Intervencionista. Hospital Universitario Carlos Haya. Málaga. España.
}

\begin{abstract}
El vasospasmo cerebral es una de las principales causas de morbimortalidad en los pacientes con hemorragia subaracnoidea por rotura de un aneurisma cerebral. Cuando el vasospasmo se hace resistente al tratamiento médico máximo, el tratamiento endovascular es una opción terapéutica para incrementar el flujo sanguíneo cerebral y evitar lesiones isquémicas cerebrales. Los rápidos avances en técnicas endovasculares permiten utilizar la angioplastia transluminal percutánea y la infusión intraarterial de diversos fármacos vasodilatadores para revertir el vasospasmo. En este artículo se revisan las diferentes técnicas endovasculares disponibles y se describen sus mecanismos de acción, técnicas de administración, resultados clínicos y complicaciones.
\end{abstract}

PALABRAS CLAVE: Vasospasmo intracraneal. Hemorragia subaracnoidea. Tratamiento endovascular. Aneurisma. Angioplastia con balón.

\section{ENDOVASCULAR TREATMENT OF CEREBRAL VASOSPASM DUE TO ANEURYSMAL SUBARACHNOID HEMORRHAGE}

Cerebral vasospasm remains a leading cause of death and disability in patients with aneurysmal subarachnoid hemorrhage. When vasospasm becomes refractory to maximal medical treatment,

Correspondencia: Dr. P.P. Alcázar Romero.

Unidad de Neurorradiología Intervencionista. Hospital Universitario

Virgen de las Nieves. Hospital de Rehabilitación y Traumatología.

Ctra. de Jaén, s/n. 18013 Granada. España.

Correo electrónico: ppalcazar@ya.com

Manuscrito aceptado el 6-6-2008. endovascular therapies may be considered as an option to increase cerebral blood flow to prevent cerebral infarction. Endovascular techniques include transluminal balloon angioplasty and intraarterial infusion of vasorelaxants.

This article reviews the various endovascular techniques for the treatment of cerebral vasospasm and discusses the mechanisms of action, techniques of administration, clinical results, and limitations of these treatment strategies.

KEY WORDS: Intracranial vasospasm. Subarachnoid hemorrhage. Endovascular therapy. Aneurysm. Angioplasty. Balloon.

\section{INTRODUCCIÓN}

El vasospasmo es la principal causa de muerte y discapacidad tras los efectos iniciales de la hemorragia subaracnoidea (HSA) causada por la rotura de un aneurisma cerebral.

A pesar de los avances introducidos en la terapia neurointensiva y el desarrollo de nuevas técnicas endovasculares, se estima que un 25-30\% de los pacientes que sobreviven a una hemorragia subaracnoidea aneurismática sufren complicaciones isquémicas en las siguientes semanas y entre el 12 y el $17 \%$ de todos los casos de HSA mueren o permanecen con gran discapacidad debido a complicaciones isquémicas ${ }^{1}$. Se estima que en un 5-9\% de los pacientes con HSA que mueren o permanecen con discapacidad severa el vasospasmo es la causa ${ }^{2-4}$.

Se define vasospasmo cerebral como el estrechamiento diferido y reversible de las arterias cerebrales que ocurre tras la HSA (típicamente entre el cuarto y 
el decimocuarto día) y afecta comúnmente a las arterias proximales del polígono de Willis. Aunque nuestro conocimiento sobre sus mecanismos fisiopatológicos es incompleto, se considera que el contacto prolongado de la sangre subaracnoidea con la pared de las arterias es el factor primario inductor del vasospasmo. Dicho estrechamiento arterial afecta al flujo sanguíneo cerebral y la adecuada oxigenación del tejido cerebral, y puede dar lugar a fenómenos isquémicos que pueden manifestarse como disminución del nivel de conciencia, estado confusional, alteraciones motrices o del lenguaje, incremento de la presión arterial y empeoramiento de la cefalea. El diagnóstico de vasospasmo se basa principalmente en la clínica neurológica y en los hallazgos en la angiografía cerebral. Aunque el vasospasmo angiográfico ocurre en el $70 \%$ de los pacientes, sólo en un 20-30\% se desarrolla un déficit neurológico isquémico tardío (delayed ischemic neurological deficit [DIND] en la literatura anglosajona $)^{3}$. Entre sus factores predictores se encuentran la presencia de un grueso coágulo cisternal, el rápido incremento en las velocidades de la arteria cerebral media según el Doppler transcraneal, una puntuación en la escala de coma de Glasgow $<14$ al ingreso y la rotura de un aneurisma de la arteria comunicante anterior o de la arteria carótida interna ${ }^{4}$.

Entre las estrategias terapéuticas utilizadas actualmente para la prevención del vasospasmo sintomático, se encuentran la llamada triple $\mathrm{H}^{5}$ (hipertensión, hipervolemia, hemodilución), la administración de antagonistas de los canales del calcio ${ }^{6}$, la cirugía precoz con retirada del coágulo subaracnoideo ${ }^{7}$ y el drenaje de líquido cefalorraquídeo en la región lumbar ${ }^{8}$.

El tratamiento endovascular se reserva típicamente para tratar los DNID resistentes al tratamiento médico máximo. Sin embargo, las indicaciones clínicas concretas y el momento más adecuado para su instauración no están bien establecidos. Ante un DNID en el que se han descartado otras causas como hidrocefalia, crisis comiciales o alteraciones electrolíticas y que no responde a la terapia triple $\mathrm{H}$, debería considerarse la terapéutica endovascular y eventualmente instaurarse horas después del inicio del cuadro deficitario. El beneficio parece ser mayor cuanto más precozmente se inicie el tratamiento, por lo que la detección temprana del DNID es de vital importancia. En pacientes con signos lateralizadores, el diagnóstico de DNID puede no ser dificultoso. Sin embargo, en pacientes con aneurismas de la línea media, que tienden a presentar cambios sutiles en el estado mental difíciles de objetivar, se ha definido DNID como una caída de más de 2 puntos en la escala de coma de Glasgow ${ }^{9}$. Eskridge et $\mathrm{al}^{10}$ han propuesto los siguientes criterios de indicación del tratamiento endovascular: $a$ ) déficit neurológico de nueva aparición no debida a otras causas; $b$ ) que no haya evidencia de infarto establecido en la tomografía computarizada (TC) craneal; $c$ ) déficit resistente al tratamiento hiperdinámico, y $d$ ) evidencia angiográfica de vasospasmo que concuerde topográficamente con la clínica deficitaria.

El objetivo del tratamiento endovascular es el incremento del flujo sanguíneo cerebral para evitar da- ño isquémico irreversible, por lo que el mayor beneficio se obtiene cuanto más precozmente se instaure. Consiste en la dilatación mecánica de arterias mediante catéteres-balón (angioplastia transluminal percutánea $[\mathrm{ATP}])$ y/o mediante la instilación intraarterial de diferentes fármacos vasodilatadores.

En este artículo se revisan las técnicas endovasculares disponibles en la actualidad para el tratamiento del vasospasmo sintomático resistente al tratamiento médico como la angioplastia transluminal con balón y la infusión intraarterial de diferentes fármacos vasodilatadores. Se discuten las indicaciones, los mecanismos de acción, la técnica de administración, la eficacia y las complicaciones potenciales de cada una de ellas.

\section{ANGIOPLASTIA TRANSLUMINAL CON BALÓN}

En 1984 Zubkov et al $^{11}$ publicaron por primera vez la utilización de angioplastia con balón para el tratamiento del vasospasmo en pacientes con HSA, y demostraron la capacidad para tratar por vía endovascular las lesiones estenóticas intracraneales. Desde entonces, numerosas publicaciones han demostrado la eficacia y la seguridad de este tratamiento ${ }^{12-18}$.

\section{Mecanismo de acción}

La ATP se ha usado para tratar de forma efectiva el vasospasmo localizado en los segmentos proximales de las arterias cerebrales compuestos por capas musculares más gruesas. El objetivo primario de la ATP es dilatar estos segmentos proximales para mejorar con ello el flujo sanguíneo, la oxigenación y el metabolismo del territorio distal.

Aunque la experiencia en humanos ha demostrado que la ATP revierte el vasospasmo de forma duradera, no se conoce completamente el mecanismo preciso por el que se produce este efecto. Los hallazgos histológicos en las arterias afectadas por vasospasmo muestran diferencias significativas entre las arterias intervenidas y las no tratadas. Éstas muestran proliferación de tejido conectivo en la íntima y en la capa media, mientras que las arterias tratadas con ATP muestran compresión y estiramiento de sus paredes (fibras musculares lisas, tejido conectivo y lámina elástica interna) sin daño celular o rotura ${ }^{13,14}$. Se postula que la disfunción de las fibras musculares y la rotura del tejido conectivo proliferante en la pared arterial vasospástica son los mecanismos por los que la ATP logra su efecto vasodilatador duradero ${ }^{15}$.

\section{Técnica de administración}

\section{Tipo de balón}

Desde la descripción inicial de Zubkov et $\mathrm{al}^{11}$ en 1984 , se han desarrollado innovaciones tecnológicas en catéteres y balones que ha conferido a la ATP más seguridad y aplicabilidad. Inicialmente se utilizaron balones de látex relativamente rígidos, que fueron sus- 
tituidos a finales de los años ochenta por balones de silicona dirigidos por flujo y, más recientemente, balones dirigidos con guía. Éstos mejoran la navegabilidad y, por lo tanto, la capacidad para cateterizar vasos inalcanzables para los dirigidos por flujo, aunque podrían conllevar mayor riesgo de perforación vascular y rotura ${ }^{16}$. Entre los balones dirigidos con guía, existen modelos flexibles y no flexibles. Los balones flexibles son teóricamente menos traumáticos para la pared del vaso y navegan más fácilmente en anatomías tortuosas. Sin embargo, sus diámetros mínimos disponibles son 3,5-4 mm, tamaño mayor que la porción más proximal de las arterias cerebrales anterior o media, lo que supone riesgo de rotura de esos vasos. Los balones no flexibles, aunque son más traumáticos por su mayor rigidez, están disponibles en tamaños de 22,25 mm y no se puede inflarlos fácilmente por encima de su diámetro nominal. Sólo desde hace poco se dispone de balones específicamente diseñados para la ATP intracraneal, que probablemente mejoren las cifras de eficacia y seguridad de esta técnica publicadas en la década pasada ${ }^{17}$. De estos balones, merece mencionar el balón flexible de baja presión dirigido con guía Hyperglide (EV3 Neurovascular, Irvine, California, Estados Unidos), así como el balón no flexible dirigido por guía Gateway (Boston Scientifics, Nanterre, Cedex, Francia).

\section{Descripción del procedimiento}

Antes de iniciar el procedimiento endovascular, se realiza una TC craneal para descartar hemorragia cerebral, hidrocefalia o infarto extenso reciente. La ATP intracraneal se lleva a cabo vía transfemoral y debería limitarse a equipos de neurorradiólogos intervencionistas con adecuado entrenamiento en estas técnicas. Inicialmente se obtienen series angiográficas con sustracción digital para dilucidar si la localización de los vasos espásticos se correlaciona con el déficit neurológico del paciente. El procedimiento terapéutico se lleva a cabo idealmente con anestesia general, para evitar movimientos indeseados del paciente que podrían favorecer complicaciones como la rotura arterial. Para evitar fenómenos tromboembólicos, se hepariniza a los pacientes, a menos que haya una contraindicación absoluta, antes de la cateterización intracraneal hasta mantener un tiempo de coagulación activado (ACT) de 2,5-3 veces el control basal o generalmente $>300 \mathrm{~s}$. Tras la colocación de un catéter guía de 5 o 6 Fr en la porción cervical distal de la arteria cerebral afectada, y controlando con mapa arterial digital (roadmap), se realiza el microcateterismo de la arteria o las arterias afectadas mediante un catéter balón, con el que se procede a la angioplastia a continuación. Típicamente el vasospasmo afecta a los segmentos $\mathrm{A}_{1}$ de la arteria cerebral anterior y a los $M_{1} y_{2} M_{2}$ de la arteria cerebral media, y menos frecuentemente a los segmentos $\mathrm{P}_{1}$ de la arteria cerebral posterior y otros vasos del territorio vertebrobasilar. Los segmentos distales de la vasculatura cerebral no se han considerado tratables mediante
ATP por la dificultad de acceso por vía endovascular, así como por cuestiones de seguridad de la dilatación transluminal en vasos de paredes finas. Puede ser necesaria la infusión intraarterial de papaverina para facilitar el paso del microcatéter por el vaso espástico. La técnica de ATP intracraneal requiere dilataciones lentamente progresivas. Eskridge et $\mathrm{al}^{10}$ recomendaban dilataciones mecánicas sucesivas con diámetros crecientes de la luz del vaso en cuatro pasos (el 25, el 50 , el 75 y el $100 \%$ del diámetro) con desinflados intermedios. Debe tenerse especial cuidado en no dilatar el vaso por encima de su diámetro original para minimizar el riesgo de rotura arterial.

\section{Eficacia clínica y resultados}

Se ha evaluado el efecto de la ATP en función de diferentes variables resultado, como son la mejoría neurológica, los cambios angiográficos y de Doppler transcraneal (DTC) o mediciones del flujo sanguíneo cerebral. Los cambios angiográficos y de velocidades en el DTC no son los marcadores ideales para valorar el éxito del tratamiento, puesto que no proporcionan información sobre el estado funcional del lecho vascular distal. Éste puede evaluarse indirectamente por la mejoría clínica o directamente mediante estudios de flujo sanguíneo cerebral antes y después del tratamiento. A pesar de que se han publicado excelentes resultados angiográficos de la ATP (mejoría angiográfica cercana al $100 \%)^{18,19}$, sus resultados clínicos son variables y las tasas de mejoría neurológica oscilan entre el 31 y el $93 \%$ tras el tratamiento $0^{20-22}$. En una extensa revisión de la literatura, Hoh et al $^{21}$ estimaron que el $62 \%$ de los pacientes mejoraron de su déficit neurológico tras la angioplastia. La probabilidad de obtener resultados clínicos favorables está relacionada con la instauración precoz del tratamiento tras la aparición del DNID, aunque las ventanas terapéuticas máximas recomendadas varían: $2 h^{22}, 12 h^{12,23}$ o $24 h^{20}$. En nuestra opinión, el tratamiento endovascular no debería demorarse más de $2 \mathrm{~h}$ tras la aparición del DNID.

Las variaciones del flujo sanguíneo cerebral tras la ATP se han medido mediante resonancia magnética (potenciada en difusión y en perfusión), TC con xenón, tomografía por emisión de positrones (PET) y TC por emisiones monofotónicas (SPECT). Hoh et $\mathrm{al}^{21}$ publicaron una mejoría general del flujo sanguíneo cerebral en el $85 \%$ de los pacientes tratados con ATP.

Por el momento no se han publicado datos de ensayos clínicos aleatorizados que evalúen el efecto de la ATP en el resultado neurológico final. Existe un ensayo clínico en marcha $-\mathrm{A}$ trial of cerebral angioplasty for post subarachnoid haemorrhage symptomatic vasospasm $\left(\right.$ VERITAS $\left.^{24}\right)$-, cuya población de estudio son pacientes con DNID tras HSA y que examina si a 1 año de seguimiento la ATP combinada con el mejor tratamiento médico reduce la proporción de pacientes con resultado neurológico desfavorable respecto al mejor tratamiento médico solo. Dicho estudio tiene prevista su finalización en 2010 tras reclutar a 200 pacientes. 
Balloon Prophylaxis of Aneurysmal Vasospasm ${ }^{25}$ es otro ensayo clínico cuyo propósito es determinar, en pacientes con HSA de grado III de Fisher, si la ATP precoz previene el desarrollo de vasospasmo y mejora el resultado neurológico (medido según la escala Glasgow Outcome Scale [GOS]) a los 6 meses. Dicho estudio, financiado por el National Institute of Neurological Disorders and Stroke (NINDS) de Estados Unidos, ha sido publicado recientemente. Sus resultados indican que la angioplastia profiláctica no mejora el resultado neurológico de los pacientes con HSA grado III de Fisher, aunque se constata, según los autores, una tendencia a que se produzcan menos casos de vasospasmo en los pacientes tratados que en el grupo control.

\section{Complicaciones relacionadas con la ATP}

La tasa de complicaciones mayores se estima en torno a un 5\%, con una incidencia de rotura arterial (una complicación casi invariablemente fatal) del $1,1 \%{ }^{21}$. Entre las complicaciones publicadas se encuentran la tromboembolia, la disección arterial, la hemorragia por reperfusión, el resangrado de aneurisma no tratado y la rotura arterial intracraneal.

\section{INFUSIÓN INTRAARTERIAL DE FÁRMACOS VASOACTIVOS}

\section{Papaverina}

El clorhidrato de papaverina es uno de los fármacos más comúnmente usados en el tratamiento del vasospasmo cerebral desde los estudios iniciales descritos por Kaku et $\mathrm{al}^{26}$ y Kassel et $\mathrm{al}^{27}$ en 1992. La papaverina es un potente vasodilatador arterial tanto proximal como distal, por lo que se lo considera indicado para el tratamiento del vasospasmo difuso y en vasculatura distal no asequible a la cateterización con balón para angioplastia. Entre sus inconvenientes se encuentran su efecto transitorio (lo que condiciona múltiples tratamientos) y el riesgo de incrementar la presión intracraneal, por lo que su utilización actual en el vasospasmo cerebral está en retroceso.

\section{Mecanismo de acción}

La papaverina es un alcaloide derivado del opio del grupo de las bencilisoquinolinas, del que actualmente se fabrica la forma sintética. Es un potente relajante de la musculatura lisa vascular no dependiente del endotelio, capaz de producir dilatación difusa de arterias, arteriolas y venas. Su mecanismo de acción no se conoce bien, aunque se presume su relación con la inhibición inespecífica de la fosfodiesterasa, una enzima intracelular que degrada el 3',5' adenosinmonofosfato cíclico (AMPc) y el 3',5' guanosinmonofosfato cíclico (GMPc), que se acumulan e interfieren los mecanismos de la transmisión necesaria para la contracción de la célula muscular lisa. Asimismo, la papaverina puede inhibir la liberación de calcio del espacio intracelu- lar mediante el bloqueo de sus canales en la membrana celular ${ }^{28}$. La papaverina tiene una corta vida media plasmática (45-60 min) ${ }^{1}$, lo que podría explicar su efecto transitorio en la vasculatura cerebral.

\section{Técnica de administración}

La papaverina intraarterial se administra superselectivamente a través de un microcatéter colocado proximal al vaso afecto. La concentración usada habitualmente es del 0,3\% (300 mg de papaverina en $100 \mathrm{ml}$ de suero fisiológico) y se infunde a un flujo de $3 \mathrm{ml} / \mathrm{min}$ en el territorio vascular afectado. La papaverina no debe mezclarse con agentes de contraste o heparina, que favorecen su precipitación en forma de microcristales que pueden ser causa de embolias ${ }^{29}$. En la circulación anterior, el microcatéter suele colocarse en la porción supraclinoidea de la arteria carótida interna, por encima del origen de la arteria oftálmica para poder infundir simultáneamente los territorios de las arterias cerebral anterior y media. En el territorio posterior, el catéter se coloca inmediatamente proximal al área afectada, aunque no se recomienda la infusión directa de papaverina en el tronco basilar por debajo del origen de la arteria cerebelosa anteroinferior, para evitar la difusión por perforantes bulbares. Durante la infusión, es importante monitorizar la presión arterial, la presión intracraneal y el estado neurológico del paciente. El tratamiento debe interrumpirse inmediatamente si se produce hipotensión, incremento de la presión intracraneal o deterioro neurológico.

\section{Resultados clínicos}

La tasa de mejoría clínica derivada de la infusión de papaverina intraarterial es modesta y oscila entre el 25 y el $52 \%$ en la mayoría de las series. En una de las revisiones de la literatura más extensas, Hoh et $\mathrm{al}^{21}$ describen mejoría clínica en el $43 \%$ de los 346 pacientes tratados. En cualquier caso, esos datos provienen de numerosas series cortas de pacientes con análisis retrospectivos, y la administración intraarterial de papaverina todavía no ha sido sometida a ensayos clínicos para demostrar su eficacia.

Existen estudios que demuestran que la infusión intraarterial de papaverina se asocia con incremento del diámetro arterial y mejoría del tiempo de circulación cerebral medidos angiográficamente ${ }^{30-34}$, con mejoría tanto del flujo sanguíneo cerebral mediante la dilatación arterial proximal, intermedia y distal ${ }^{30}$ como de la oxigenación cerebral ${ }^{31}$.

\section{Complicaciones}

La tasa global de complicaciones derivadas de la infusión intraarterial de papaverina se estima en el $9,9 \%$ de los $\operatorname{casos}^{21}$. Entre ellas se incluyen elevación de la presión intracraneal, convulsiones, hemiparesia transitoria, midriasis, hipotensión, disfunción cardíaca y parada respiratoria ${ }^{1}$. La infusión proximal a la ar- 
teria oftálmica se asocia con midriasis, que habitualmente es transitoria, pero se han descrito casos de deterioro visual y ceguera monocular ${ }^{30}$. La infusión en la circulación posterior puede dar lugar a disfunción cardíaca y parada respiratoria, probablemente como resultado de la depresión de los centros respiratorio y cardiovascular en el tronco cerebral. La causa de síntomas neurológicos como hemiparesia o convulsiones no es conocida. Se postula que la formación de microcristales en las zonas de bajo flujo por precipitación de la papaverina podría originar embolias distales. Se podría atribuir las crisis comiciales a un posible efecto proconvulsivo de la papaverina a altas dosis o a un efecto neurotóxico directo ${ }^{1}$.

Una de las complicaciones más graves es el rápido incremento de la presión intracraneal atribuible a la elevación del volumen y el flujo sanguíneos cerebrales tras la vasodilatación cerebral. La hipertensión intracraneal puede ser intratable y puede desencadenar la muerte del paciente. Esta complicación aparece hasta en el $10 \%$ de los pacientes tratados ${ }^{32}$.

\section{BLOQUEADORES DE LOS CANALES DEL CALCIO}

Debido a los inconvenientes descritos en la utilización intraarterial de papaverina para el tratamiento del vasospasmo cerebral sintomático, los bloqueadores de los canales del calcio, como nimodipino, nicardipino y verapamilo, se han considerado una alternativa a aquélla en esta particular indicación.

$\mathrm{Su}$ mecanismo de acción se basa en la reducción de la entrada de iones calcio a la célula muscular lisa a través de los canales del calcio tipo L. La reducción intracelular de iones de calcio libres causa la interrupción del complejo actina-miosina, imprescindible para la contracción. Además de la relajación de la musculatura lisa vascular, condicionan efectos cardíacos como el bloqueo de los nodos sinoatrial y atrioventricular y tienen efecto inotrópico negativo. El nicardipino es el que menos efecto tiene en el funcionamiento cardíaco, seguido del nimodipino y el verapamilo, que muestra potente efecto inotrópico negativo e inhibidor de la conducción cardíaca.

Debido a su capacidad para relajar la célula muscular lisa vascular, se realizaron ensayos clínicos con nimodipino y nicardipino, administrados profilácticamente por vía oral o intravenosa, para evaluar si reducían la proporción de pacientes con mal resultado neurológico y DNID tras $\mathrm{HSA}^{33-39}$. De ellos, solamente el nimodipino produjo una modesta mejoría en el resultado neurológico, por lo que es uno de los fármacos más utilizados por vía intraarterial. Ambos previenen la isquemia secundaria tras $\mathrm{HSA}^{34,35}$.

\section{Nimodipino}

El nimodipino para administración parenteral contiene como excipiente alcohol etílico, que puede ser tóxico en la circulación vascular. Se prepara diluyendo 1-3 $\mathrm{mg}$ en 15-45 $\mathrm{ml}$ de suero fisiológico y se in- funde lentamente en $10-30 \mathrm{~min}(0,1 \mathrm{mg} / \mathrm{min})$ en el territorio arterial afectado, tras cateterización superselectiva (figs. 1 y 2). La dosis total depende del grado inicial del vasospasmo, el grado de respuesta angiográfica obtenida y la presión arterial inicial, aunque no debe superar los $5 \mathrm{mg}$ por procedimiento ${ }^{36}$. Durante la infusión deben monitorizarse el estado neurológico del paciente, la presión arterial y la presión intracraneal.

Existen dos pequeños estudios retrospectivos que evalúan la seguridad y la eficacia de la administración intraarterial de nimodipino ${ }^{37,38}$. En ambos se constata una mejoría clínica inicial de un 76-89\% y dilatación angiográfica en un 43-69\%, y no se detectan complicaciones mayores (empeoramiento del vasospasmo, hipotensión prolongada o incremento de la presión intracraneal).

\section{Nicardipino}

El nicardipino ha demostrado su eficacia para prevenir el vasospasmo en otras formas de administración como la infusión intratecal y la colocación de implantes de liberación lenta en el espacio subaracnoideo ${ }^{39,40}$. Para su administración intraarterial, se diluye en suero salino hasta una concentración de 0,1 $\mathrm{mg} / \mathrm{ml}$ y se infunde, vía microcateterización superselectiva del territorio arterial afectado, en dosis sucesivas de $1 \mathrm{ml}$ hasta un máximo de $5 \mathrm{mg}$ por vaso ${ }^{41}$. Badjatia et $\mathrm{al}^{41}$ aplicaron este tratamiento a 18 pacientes, y consiguieron dilatación angiográfica y por DTC en todos los vasos tratados y mejoría neurológi-

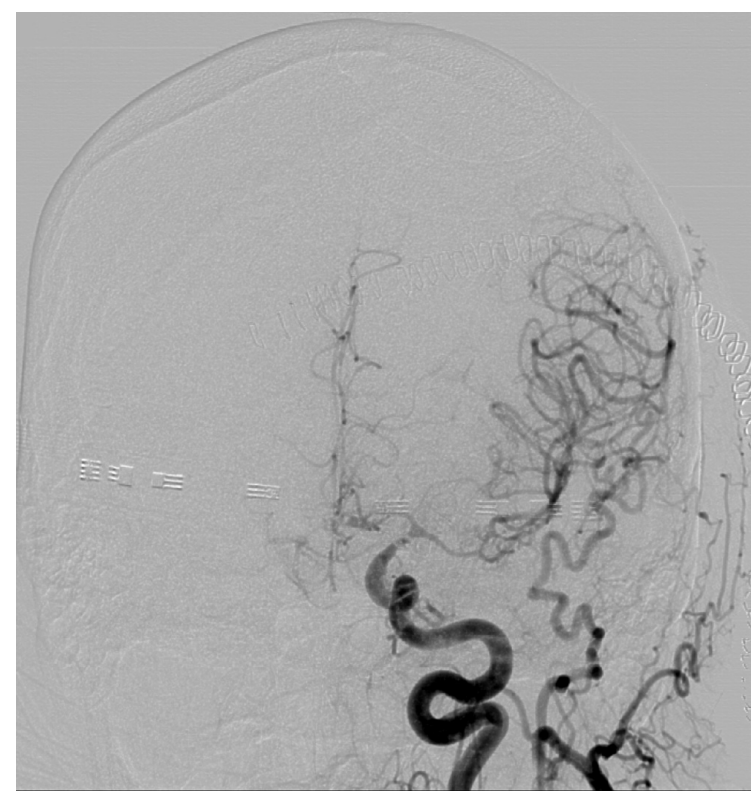

Figura 1. Angiografía carotídea izquierda en proyección anteroposterior que muestra vasospasmo severo que afecta a la porción supraclinoidea de la arteria carótida interna, así como a los segmentos $A_{1}$ y $M_{1}$ en paciente con hemorragia subaracnoidea por rotura de aneurisma de la arteria comunicante anterior tratado quirúrgicamente. 


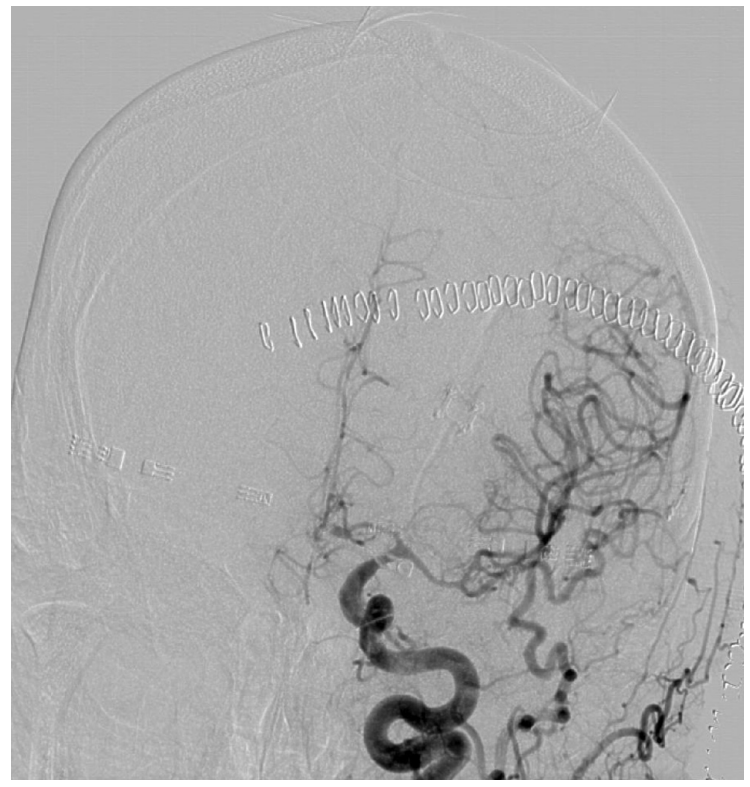

Figura 2. Arteriografía carotídea izquierda tras la infusión intraarterial superselectiva de $2 \mathrm{mg}$ de nimodipino en segmentos $A_{1} y$ $M_{l}$ y carótida supraclinoidea. Se aprecia un moderado incremento de calibre en los territorios arteriales tratados.

ca en el $42 \%$ de los pacientes. Sólo 1 paciente sufrió recurrencia del vasospasmo que necesitó un tratamiento adicional. La complicación mayor publicada por este grupo consistió en incremento de la presión intracraneal que requirió la apertura de la ventriculostomía en el 33\% de los pacientes, por lo que la monitorización de la presión intracraneal con este tratamiento es de vital importancia.

\section{Verapamilo}

El verapamilo se ha utilizado extensamente para el tratamiento del vasospasmo coronario y ocasionalmente en el cerebro para revertir el vasospasmo debido a la colocación de catéteres guía y balones de angioplastia. Feng et $\mathrm{al}^{42}$ administraron verapamilo en bolos intraarteriales de 1-2 mg, y en su estudio retrospectivo de 29 pacientes no encontraron efectos adversos significativos cardiovasculares (hipotensión o bradicardia) ni signos clínicos de incremento de la presión intracraneal, por lo que concluyeron que su uso intraarterial es seguro para el cerebro. No hay datos acerca de la eficacia del verapamilo en el contexto del vasospasmo por HSA.

\section{OTROS FÁRMACOS VASODILATADORES}

Otros fármacos vasodilatadores se han usado de forma anecdótica para la irrigación intraarterial de la circulación cerebral en el tratamiento del vasospasmo sintomático por HSA.

El fasudilo es un fármaco vasodilatador con relativa especificidad para la vasculatura cerebral. En un ensayo clínico ${ }^{43}$ publicado en 1992 se demostró que su administración intravenosa reduce significativamente el vasospasmo angiográfico y sintomático en pacientes con HSA. Se han publicado dos estudios sobre su uso intraarterial en un número de pacientes pequeño ${ }^{44,45}$, con mejorías clínicas y angiográficas del $65 \%$ y mínimos efectos adversos (hipotensión y alteración del nivel de conciencia transitorio).

La amrinona y la colforsina daropato son fármacos vasodilatadores coronarios con diferentes mecanismos de acción, ensayados por vía intraarterial en la circulación cerebral para revertir el vasospasmo por HSA. La experiencia con estos fármacos se limita a $7^{46}$ y 2 pacientes ${ }^{47}$, con resultados positivos en cuanto a seguridad y eficacia.

\section{CONCLUSIONES}

Las terapias endovasculares ofrecen oportunidades de revertir el vasospasmo cerebral por hemorragia subaracnoidea en pacientes resistentes al tratamiento médico máximo. La angioplastia transluminal percutánea permite la dilatación de las arterias proximales de mayor tamaño, pero su capacidad para alcanzar las arterias distales de menor calibre es escasa. Se ha demostrado que la papaverina intraarterial es efectiva para dilatar tanto la vasculatura distal como la proximal, pero su efecto transitorio y el riesgo de aumento de la presión intracraneal limitan su uso. Otros fármacos vasodilatadores muestran resultados preliminares favorables por vía intraarterial.

La contribución de estudios aleatorizados será necesaria para establecer el papel definitivo de las terapias endovasculares en el tratamiento del vasospasmo por HSA.

\section{BIBLIOGRAFÍA}

1. Zwienenberg-Lee M, Hartman J, Rudisil N, Muizelaar JP. Endovascular management of cerebral vasospasm. Neurosurgery. 2006;59 Suppl 3:S139-47.

2. Dehdashti AR, Mermillod B, Rufenacht DA, Reverdin A, De Tribolet N. Does treatment modality of intracranial ruptured aneurysms influence the incidence of cerebral vasospasm and clinical outcome? Cerebrovasc Dis. 2004;17:53-60.

3. Kassell NF, Sasaki T, Colohan AR, Nazar G. Cerebral vasospasm following aneurysmal subarachnoid hemorrhage. Stroke. 1985; 16:562-72.

4. Qureshi AI, Sung GY, Razumovsky AY, Lane K, Straw RN, Ulatowski JA. Early identification of patients at risk for symptomatic vasospasm after aneurysmal subarachnoid hemorrhage. Crit Care Med. 2000;28:984-90.

5. Awad IA, Carter LP, Spetzler RF, Medina M, Williams FC. Clinical vasospasm after subarachnoid hemorrhage: response to hypervolemic hemodilution and arterial hypertension. Stroke. 1987; 18:365-72.

6. Feigin VL, Rinkel GJ, Algra A, Vermeulen M, Van Gijn J. Calcium antagonists in patients with aneurysmal subarachnoid hemorrhage: a systematic review. Neurology. 1998;50:876-83.

7. Handa Y, Weir BK, Nosko M, Mosewich R, Tsuji T, Grace $\mathrm{M}$. The effect of timing of clot removal on chronic vasospasm in a primate model. J Neurosurg. 1987;67:558-64.

8. Klimo P Jr, Kestle JR, MacDonald JD, Schmidt RH. Marked reduction of cerebral vasospasm with lumbar drainage of cerebrospinal fluid after subarachnoid hemorrhage. J Neurosurg. 2004;100:215-24. 
9. Muizelaar JP, Zwienenberg M, Rudisill NA, Hecht ST. The prophylactic use of transluminal balloon angioplasty in patients with Fisher Grade 3 subarachnoid hemorrhage: A pilot study. J Neurosurg. 1999;91:51-8

10. Eskridge JM, McAuliffe W, Song JK, Deliganis AV, Newell DW, Lewis DH, et al. Balloon angioplasty for the treatment of vasospasm: results of first 50 cases. Neurosurgery. 1998;42:510-7.

11. Zubkov YN, Nikiforov BM, Shustin VA. Balloon catheter technique for dilatation of constricted cerebral arteries after aneurysmal SAH. Acta Neurochir (Wien). 1984;70:65-79.

12. Barnwell SL, Higashida RT, Halbach VV, Dowd CF, Wilson CB, Hieshima GB. Transluminal angioplasty of intracerebral vessels for cerebral arterial spasm: Reversal of neurological deficits after delayed treatment. Neurosurgery. 1989;25:424-9.

13. Honma Y, Fujiwara T, Irie K, Ohkawa M, Nagao S Morphological changes in human cerebral arteries after percutaneous transluminal angioplasty for vasospasm caused by subarachnoid hemorrhage. Neurosurgery. 1995;36:1073-81.

14. Zubkov AY, Lewis AI, Scalzo D, Bernanke DH, Harkey HL. Morphological changes after percutaneous transluminal angioplasty. Surg Neurol. 1999:51:399-403.

15. Yamamoto Y, Smith RR, Bernanke DH. Mechanism of action of balloon angioplasty in cerebral vasospasm. Neurosurgery. 1992;30:1-6.

16. Newell DW, Eskridge JM, Mayberg MR, Grady MS, Winn HR. Angioplasty for the treatment of symptomatic vasospasm following subarachnoid hemorrhage. J Neurosurg. 1989;71:654-60

17. Terry A, Zipfel G, Milner E, Cross DW, Moran C, Diringer $\mathrm{M}$, et al. Safety and technical efficacy of over-the-wire balloons for the treatment of subarachnoid hemorrhage-induced cerebral vasospasm. Neurosurg Focus. 2006;21:E14.

18. Firlik AD, Kaufmann AM, Jungreis CA, Yonas H. Effect of transluminal angioplasty on cerebral blood flow in the management of symptomatic vasospasm following aneurysmal subarachnoid hemorrhage. J Neurosurg. 1997;86:830-9.

19. Murai Y, Kominami S, Kobayashi S, Mizunari T, Teramoto A. The long-term effects of transluminal balloon angioplasty for vasospasms after subarachnoid hemorrhage: analyses of cerebral blood flow and reactivity. Surg Neurol. 2005;64:122-7.

20. Bejjani GK, Bank WO, Olan WJ, Sekhar LN. The efficacy and safety of angioplasty for cerebral vasospasm after subarachnoid hemorrhage. Neurosurgery. 1998;42:979-87.

21. Hoh BL, Ogilvy CS. Endovascular treatment of cerebral vasospasm: transluminal balloon angioplasty, intra-arterial papaverine, and intra-arterial nicardipine. Neurosurg Clin North Am. 2005; 16:501-16.

22. Rosenwasser RH, Armonda RA, Thomas JE, Benitez RP, Gannon PM, Harrop J. Therapeutic modalities for the management of cerebral vasospasm: timing of endovascular options. Neurosurgery. 1999;44:975-80.

23. Newell DW, Elliott JP, Eskridge JM, Winn HR. Endovascular therapy for aneurysmal vasospasm. Crit Care Clin. 1999;15:685-99.

24. A trial of cerebral angioplasty for post subarachnoid haemorrhage symptomatic vasospasm: UK Clinical Trials Gateway [base de datos en internet] [citado 8 May 2008]. Disponible en: http://www.controlled-trials.com/mrct/trial/240537/VERITAS

25. Zwienenberg-Lee M, Hartman J, Rudisill N, Madden LK, Smith K, Eskridge J, et al; The Balloon Prophylaxis for Aneurysmal Vasospasm (BPAV) Study Group. Effect of prophylactic transluminal balloon angioplasty on cerebral vasospasm and outcome in patients with Fisher grade III subarachnoid hemorrhage. Results of a phase II multicenter, randomized, clinical trial. Stroke. 2008;39:1759-65.

26. Kaku Y, Yonekawa Y, Tsukahara T, Kazekawa K. Superselective intra-arterial infusion of papaverine for the treatment of cerebral vasospasm after subarachnoid hemorrhage. J Neurosurg. 1992;77:842-7.

27. Kassell NF, Helm G, Simmons N, Phillips CD, Cail WS. Treatment of cerebral vasospasm with intra-arterial papaverine. J Neurosurg. 1992;77:848-52.

28. Newell DW, Elliott JP, Eskridge JM, Winn HR. Endovascular therapy for aneurysmal vasospasm. Crit Care Clin. 1999;15:685-99.
29. Oskouian RJ Jr, Martin NA, Lee JH, Glenn TC, Guthrie D, Gonzalez NR, et al. Multimodal quantitation of the effects of endovascular therapy for vasospasm on cerebral blood flow, transcranial doppler ultrasonographic velocities, and cerebral artery diameters. Neurosurgery. 2002;51:30-43.

30. Clouston JE, Numaguchi Y, Zoarski GH, Aldrich EF, Simard JM, Zitnay KM. Intraarterial papaverine infusion for cerebral vasospasm after subarachnoid hemorrhage. AJNR Am J Neuroradiol. 1995;16:27-38.

31. Fandino J, Kaku Y, Schuknecht B, Valavanis A, Yonekawa Y. Improvement of cerebral oxygenation patterns and metabolic validation of superselective intraarterial infusion of papaverine for the treatment of cerebral vasospasm. J Neurosurg. 1998;89:93-100.

32. Andaluz N, Tomsick TA, Tew JM Jr, Van Loveren HR, Yeh HS, Zuccarello M. Indications for endovascular therapy for refractory vasospasm after aneurysmal subarachnoid hemorrhage: Experience at the University of Cincinnati. Surg Neurol. 2002;58:131-8.

33. Allen GS, Ahn HS, Preziosi TJ, Battye R, Boone SC, Boone $\mathrm{SC}$, et al. Cerebral arterial spasm -a controlled trial of nimodipine in patients with subarachnoid hemorrhage. N Engl J Med. 1983; 308:619-24.

34. Feigin VL, Rinkel GJ, Algra A, Vermeulen M, Van Gijn J. Calcium antagonists in patients with aneurysmal subarachnoid hemorrhage: a systematic review. Neurology. 1998;50:876-83.

35. Pickard JD, Murray GD, Illingworth R, Shaw MD, Teasdale GM, Foy PM, et al. Effect of oral nimodipine on cerebral infarction and outcome after subarachnoid haemorrhage: British Aneurysm Nimodipine Trial. BMJ. 1989;298:636-42.

36. Sayama CM, Liu JK, Couldwell WT. Update on endovascular therapies for cerebral vasospasm induced by aneurysmal subarachnoid hemorrhage. Neurosurg Focus. 2006;21:E12.

37. Biondi A, Ricciardi G, Puybasset L. Intra-arterial nimodipine for the treatment of symptomatic cerebral vasospasm after aneurysmal subarachnoid hemorrhage. AJNR Am J Neuroradiol. 2004;25:1067-76.

38. Hui C, Lau KP. Efficacy of intra-arterial nimodipine in the treatment of cerebral vasospasm complicating subarachnoid haemorrhage. Clin Radiol. 2005;60:1030-6.

39. Kasuya H, Onda H, Sasahara A, Takeshita M, Hori T. Application of nicardipine prolonged-release implants: analysis of 97 consecutive patients with acute subarachnoid hemorrhage. Neurosurgery. 2005;56:895-902.

40. Suzuki M, Doi M, Otawara Y, Ogasawara K, Ogawa A. Intrathecal administration of nicardipine hydrochloride to prevent vasospasm in patients with subarachnoid hemorrhage. Neurosurg Rev. 2001;24:180-4.

41. Badjatia N, Topcuoglu MA, Pryor JC, Rabinov JD, Ogilvy CS, Carter BS, et al. Preliminary experience with intra-arterial nicardipine as a treatment for cerebral vasospasm. AJNR Am J Neuroradiol. 2004;25:819-26.

42. Feng L, Fitzsimmons BF, Young WL, Berman MF, Lin E, Aagaard BD, et al. Intraarterially administered verapamil as adjunct therapy for cerebral vasospasm: safety and 2-year experience. AJNR Am J Neuroradiol. 2002;23:1284-90.

43. Shibuya M, Suzuki Y, Sugita K, Saito I, Sasaki T, Takakura $\mathrm{K}$, et al. Effect of AT877 on cerebral vasospasm after aneurysmal subarachnoid hemorrhage. Results of a prospective placebo controlled double-blind trial. J Neurosurg. 1992;76:571-7.

44. Tachibana E, Harada T, Shibuya M, Saito K, Takayasu M, Suzuki Y, et al. Intra-arterial infusion of fasudil hydrochloride for treating vasospasm following subarachnoid haemorrhage. Acta Neurochir (Wien). 1999;141:13-9.

45. Tanaka K, Minami H, Kota M, Kuwamura K, Kohmura E. Treatment of cerebral vasospasm with intra-arterial fasudil hydrochloride. Neurosurgery. 2005;56:214-23.

46. Arakawa Y, Kikuta K, Hojo M, Goto Y, Ishii A, Yamagata $\mathrm{S}$. Milrinone for the treatment of cerebral vasospasm after subarachnoid hemorrhage: report of seven cases. Neurosurgery. 2001;48:723-30

47. Suzuki S, Ito O, Sayama T, Yamaguchi S, Goto K, Sasaki T. Intraarterial injection of colforsin daropate hydrochloride for the treatment of vasospasm after aneurysmal subarachnoid hemorrhage: preliminary report of two cases. Neuroradiology. 2006;48:50-3. 